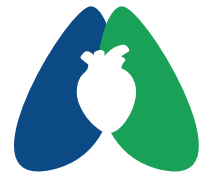

ASSOBRAFIR

CI Ê N C I A

\section{Protocolo fisioterapêutico com base na escala Perme Intensive Care Unit Mobility Score para doentes críticos}

\author{
Physiotherapeutic protocol based the Perme Scale Intensive Care \\ Unit mobility score for critical patients
}

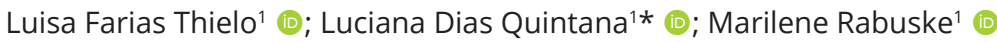

\section{Resumo}

Introdução: a mobilização precoce é capaz de prevenir e tratar complicações de saúde, assim favorecendo a condição clínica do paciente, reduzindo o tempo de internação e custos hospitalares. O foco principal do fisioterapeuta deve ser voltado para minimizar o declínio funcional durante a internação hospitalar, o que só se torna possível através de avaliações e reavaliações durante todo o período de hospitalização. Objetivo: revisar a literatura a fim de elaborar um protocolo baseado na escala Perme e no MRC para fornecer recomendações de exercícios eficazes e aplicáveis à realidade e disponibilizar um recurso para padronizar o atendimento ao paciente crítico após alta da unidade intensiva. Métodos: a pesquisa de literatura foi realizada em busca de estudos nas bases de dados eletrônicos, excluindo aqueles que não tinham relação com o tema e incluindo estudos sobre avaliação e protocolo de exercícios. Sendo assim foram utilizados 8 artigos para a elaboração do protocolo. Resultado: protocolo elaborado dividido em 4 níveis com base na escala Perme e Medical Research Council. Conclusão: avaliações periódicas e reavaliações são de grande importância para quantificar a recuperação do paciente. A mobilização precoce realizada através de um instrumento norteador como o protocolo está associada a melhores resultados funcionais. Outros estudos devem ser feitos a fim de ampliar conhecimento e quantificar maior número de evidências e resultados.

Palavras-chave: Serviço Hospitalar de Fisioterapia; Fisioterapia Intensiva; Avaliação Funcional; Força Muscular; Debilidade Muscular.

\begin{abstract}
Introduction: early mobilization is able to prevent and treat health complications, thus favoring the patient's clinical condition, reducing hospitalization time and hospital costs. The physiotherapist's main focus should be on minimizing functional decline during hospitalization, which is only possible through assessments and reassessments throughout the hospitalization period. Objective: to review the literature in order to develop a protocol based on the Perme scale and the MRC to provide recommendations for effective exercises applicable to reality and provide a resource to standardize care for critical patients after discharge from the intensive care unit. Research strategy: the literature search was carried out in search of studies in the electronic databases, excluding those that were not related to the topic. Selection criteria: Studies on evaluation and exercise protocol were included. Thus, 8 articles were used for the elaboration of the protocol. Results: protocol developed divided into 4 levels based on the Perme and Medical Research Council scale. Conclusion: Periodic evaluations and reevaluations are of great importance to quantify the patient's recovery. Early mobilization performed using a guiding instrument such as the protocol is associated with better functional results. Further studies should be carried out in order to expand knowledge and quantify a greater number of evidences and results.
\end{abstract}

Keywords: Hospital Physiotherapy Service; Intensive Physiotherapy; Functional Assessment; Muscle Strength; Muscle Weakness.

Submissão em: Dezembro 03, 2020

Aceito em: Janeiro 24, 2021

Estudo realizado em: Universidade Católica de Pelotas, Pelotas, RS, Brasil

Aprovação ética: 34566920.0.0000.5339

*Autor correspondente:

Luciana Dias Quintana. E-mail:

lucianadiasq92@hotmail.com

Este é um artigo publicado em acesso aberto (Open Access) e distribuído sob a licença Creative Commons Attribution NonComercial ShareAlike License, que permite uso, distribuição e reprodução em qualquer meio, sem restrições desde que sem fins comerciais e que o trabalho original seja corretamente citado e de forma que não indique endosso ao trabalho feito. A 


\section{INTRODUÇÃO}

Antigamente o repouso no leito era bem visto para recuperação dos pacientes críticos, porém essa conduta acarretava em maior tempo de internação e diminuição na capacidade funcional ${ }^{1}$. A mobilização precoce é uma terapia capaz de prevenir e tratar complicações de saúde como atelectasias, úlceras de pressão, atrofias, fraqueza muscular e esquelética, assim favorecendo a condição clínica do paciente, reduzindo o tempo de internação e custos hospitalares ${ }^{2}$. Com isso, os números de altas das unidades de terapia intensiva (UTI) aumentaram, gerando a importância de continuar com o cuidado e atenção ao tratamento do paciente gravemente doente fora do ambiente da UTI. O fisioterapeuta é o profissional responsável pela implantação do plano de mobilização, prescrição de exercícios e progressão deste plano em conjunto com a equipe ${ }^{3}$, tem como objetivo primordial restabelecer a independência física, e recuperar a capacidade funcional ${ }^{4}$.

Com base na importância da mobilização dessa população, bem como a carência de protocolos que possam ser utilizados nesses pacientes, é de grande importância formular um protocolo de exercícios para direcionar os fisioterapeutas em suas condutas diárias, esse trabalho contribuirá para a melhora do paciente, assim como para novas pesquisas e para tomadas de decisões quanto ao adequado manejo desses pacientes ${ }^{4}$. Portanto, o objetivo do estudo é revisar a literatura a fim de elaborar um protocolo baseado na escala Perme e no Medical Research Council (MRC) para fornecer recomendações de exercícios eficazes e aplicáveis à realidade e disponibilizar um recurso para padronizar o atendimento ao paciente crítico após alta da unidade intensiva.

\section{MÉTODOS}

Realizou-se uma revisão integrativa utilizando-se os descritores presentes no título, resumo ou assunto: fisioterapia, serviço hospitalar de fisioterapia, fisioterapia intensiva, hospitalização, força muscular, debilidade muscular, deambulação precoce e reabilitação funcional. Foram excluídos artigos sem relação com o tema de avaliação funcional e protocolos de exercícios terapêuticos, dissertações e teses acadêmicas.

A amostra (Tabela 1) foi constituída por 8 artigos das bases de dados compostas por Sistema Online de Busca e Análise de Literatura Médica (MEDLINE), Literatura Latino Americana e do Caribe em Ciências da Saúde (LILACS) e Scientific Electronic Library Online (SciELO), no período de agosto a novembro de 2020.

\section{RESULTADOS}

O protocolo se torna um instrumento norteador ao realizar o atendimento fisioterapêutico, porém antes de iniciá-lo, deve-se realizar a avaliação do doente e verificar se o paciente apresenta condições para a realização do exercício proposto, existem critérios de contraindicação e interrupção do protocolo, como instabilidade hemodinâmica, traumatismo craniano grave sem monitorização da pressão intracraniana, choque cardiogênico, após 2 horas de início da hemodiálise, ocorrência de acidente vascular encefálico, infarto agudo do miocárdio, hipertensão intracraniana, hipoglicemia, prescrição de repouso no leito, quadro hemorrágico agudo ou não identificado, novo quadro arrítmico, evitar mobilização até duas horas após aumento da dose de vasopressor $^{9}$, assim como deve-se ter atenção quando o paciente estiver com pressão arterial média ( $\mathrm{mmhg})<65$, frequência cardíaca (bpm) $<50$, saturação periférica de oxigênio $\left(\mathrm{SpO}_{2}\right)<85 \%$, frequência respiratória $(\mathrm{rpm})>35$, temperatura $\left({ }^{\circ} \mathrm{C}\right)>38^{8,11}$.

O protocolo (Tabela 2) foi elaborado contendo 4 níveis com base na pontuação máxima de 32 pontos da Escala Perme Intensive Care Unit Mobility Score (Perme), quando elevada é indicativo de alta mobilidade e menor necessidade de assistência e uma pontuação baixa indicativo de baixa mobilidade e maior necessidade de assistência7, e nos 5 níveis de força obtido para cada grupo muscular através do MRC, que avalia os quatro membros com uma pontuação de 0-5 para cada grupo, classificando como 0 nenhuma contração visível e 5 força normal. A escala considera força normal o MRC igual a 60 e fraqueza muscular o $\mathrm{MRC} \leq 48^{12}$.

\section{DISCUSSÃO}

A avaliação e identificação de distúrbios cinéticofuncionais presentes durante a internação é de fundamental importância para elaboração e progressão de um plano terapêutico adequado para cada paciente. O foco principal do fisioterapeuta deve ser voltado para minimizar o declínio funcional durante a internação hospitalar, preservar a funcionalidade do paciente e sua qualidade de vida, através de avaliações e reavaliações durante todo o período de hospitalização. Identificar a contribuição dos recursos e tratamentos desses indivíduos irá indicar melhores desfechos na recuperação do paciente ${ }^{13}$.

A mobilização precoce em doentes graves tem a finalidade de manter, restaurar e aumentar a força muscular, capacidade cardiorrespiratória, e a função física/ funcional do paciente. A técnica, assim como o protocolo criado, inclui atividades terapêuticas progressivas, tais como exercícios de mobilidade no leito, sentado na beira do leito, transferência para poltrona, ortostase e deambulação ${ }^{14}$.

Os protocolos de mobilização vão desde exercícios de menor taxa metabólica como trocas de decúbito, mobilizações passivas, ativo-assistidas, ativas, realização de transferências, sentar, levantar, até exercícios com carga para membros superiores e inferiores, utilização de cicloergômetro e deambulação sempre que possível 4,5,15. 
Tabela 1. Artigos essenciais para o estudo.

\begin{tabular}{|c|c|c|}
\hline Autores & Objetivo do estudo & Resultados \\
\hline Morris et al. ${ }^{5}$ & $\begin{array}{l}\text { Avaliar se um protocolo de mobilidade aumentava a } \\
\text { proporção de pacientes da UTI receber fisioterapia. }\end{array}$ & $\begin{array}{l}\text { O protocolo de mobilidade aplicado pela fisioterapia foi } \\
\text { viável, seguro, não aumentou os custos e foi associado } \\
\text { à redução do tempo de internação. }\end{array}$ \\
\hline França et al. ${ }^{6}$ & $\begin{array}{l}\text { Fornecer recomendações mínimas, aplicáveis à } \\
\text { realidade brasileira, sobre a fisioterapia na UTI. }\end{array}$ & $\begin{array}{c}\text { É necessária a padronização dos recursos para } \\
\text { o processo de decisão clínica e a definição mais } \\
\text { detalhada do perfil do profissional fisioterapeuta na } \\
\text { UTI. }\end{array}$ \\
\hline Perme et al. ${ }^{7}$ & $\begin{array}{c}\text { Descrever o desenvolvimento desta nova ferramenta } \\
\text { para avaliar o estado de mobilidade de um paciente, } \\
\text { examinar a confiabilidade inicial da ferramenta e } \\
\text { abordar a aplicação clínica. }\end{array}$ & $\begin{array}{c}\text { O estudo sugere que a ferramenta tem a confiabilidade } \\
\text { alta e uso clínico aceitável. }\end{array}$ \\
\hline Kawaguchi et al. ${ }^{8}$ & $\begin{array}{c}\text { Realizar a tradução e a validação cultural para a } \\
\text { língua portuguesa e determinar a concordância e } \\
\text { confiabilidade da Perme. }\end{array}$ & $\begin{array}{c}\text { As versões em português foram traduzidas e validadas, } \\
\text { seguindo diretrizes rígidas, podem ser utilizadas no } \\
\text { Brasil, apresentando confiabilidade e concordância } \\
\text { excelente entre os avaliadores. }\end{array}$ \\
\hline Dubb et al. ${ }^{9}$ & $\begin{array}{l}\text { Identificar barreiras à mobilização precoce e discutir } \\
\text { estratégias para superar essas barreiras. }\end{array}$ & $\begin{array}{c}\text { Para superar as barreiras identificadas, mais de } 70 \\
\text { estratégias foram relatadas e são sintetizadas nesta } \\
\text { revisão. }\end{array}$ \\
\hline Aquim et al. ${ }^{10}$ & $\begin{array}{l}\text { Elaborar um documento com recomendações baseadas } \\
\text { em níveis de evidência sobre a mobilização precoce, } \\
\text { com impacto positivo no atendimento aos pacientes. }\end{array}$ & $\begin{array}{l}\text { Foram encontradas evidências suficientes para a } \\
\text { realização da mobilização precoce de forma segura } \\
\text { e bem definida, com indicadores prognósticos que } \\
\text { evidenciam e recomendam a técnica. A mobilização } \\
\text { precoce está associada a melhores resultados } \\
\text { funcionais, devendo ser realizada sempre que indicada. } \\
\text { É segura, deve ser meta de toda equipe multidisciplinar. }\end{array}$ \\
\hline
\end{tabular}

Uma pontuação de 0 a 6 obtida através da Escala Perme corresponde a um paciente não responsivo ou letárgico com presença de potenciais barreiras à mobilidade como dispositivos de suporte ventilatório, acessos e infusão endovenosa, necessita de máxima assistência pois apresenta déficit funcional ${ }^{7}$. O diagnóstico de fraqueza muscular adquirida pode estar associado ao tempo de ventilação mecânica (VM) e estadia no hospital ${ }^{16}$. Com isso, é de extrema importância manter/restaurar a mobilidade do paciente, pois o imobilismo a partir de 7 a 15 dias provoca alterações musculares e respiratórias no corpo humano, principalmente no sistema musculoesquelético, como resultado de alterações do sistema ósseo, articular e muscular, assim pode acarretar em complicações como acúmulo de secreções, contratura articular, hipotrofia, atrofia muscular e osteoporose, além de úlceras de pressão e perda diária de força muscular, fatores que podem complicar a trajetória clínica do paciente se não revertidos ${ }^{17}$.

Uma boa abordagem fisioterapêutica precisa ser precedida por avaliações objetivas ${ }^{15}$, assim como revisões no prontuário e contato com a equipe multidisciplinar do paciente. A primeira terapêutica adotada deve ser o posicionamento funcional, realizado de forma passiva ou ativa através de mudanças de decúbito e alinhamento corporal, tendo efeito sobre o controle neuromusculoesquelético, minimizando os efeitos deletérios da imobilização prolongada no leito. Logo a mobilização deve ser iniciada precocemente como parte do plano terapêutico para o doente crítico. Na fase aguda, nos pacientes incapazes de movimentar-se, a mobilização precoce é a conduta adotada, que terá como objetivo a manutenção das amplitudes de movimento articular, da força muscular e prevenção de encurtamentos musculares, além de prevenir úlceras de pressão ${ }^{18}$.

Recursos adicionais como a utilização da estimulação elétrica neuromuscular (EENM), podem ser eficazes quando associados a exercícios, pois a EENM busca melhorar a capacidade de realizar exercícios aumentando a força muscular, essa técnica combinada se apresenta como um auxílio na produção de contração de um músculo, o que gera ganhos também nos mecanismos reflexos necessários para a reorganização da atividade motora, é um recurso utilizado mais na UTI pela taxa metabólica que a técnica exige, mas quando utilizado na prática terapêutica refere em melhores condições de alta do paciente ${ }^{19}$.

Um escore obtido entre 7 a 11 pontos indica um paciente acordado e alerta, que apresenta mobilidade diminuída e necessita de máxima assistência para realizar as transferências ${ }^{7}$. As avaliações visam um desfecho melhor para o paciente pois permitem traçar um planejamento terapêutico adequado quanto às necessidades de cada paciente, o trabalho fisioterapêutico conta com o objetivo de ganhar resistência e estabilidade, assim como recuperar a autonomia o mais breve possível, com progressão dos exercícios ${ }^{20}$.

A habilidade de realizar as transferências deve ganhar atenção e ser recuperada brevemente pois permitirá a 
Tabela 2. Protocolo de exercícios fisioterapêuticos para pacientes críticos.

Perme: 0-6 pontos (Paciente não responsivo ou letárgico)

- Posicionamento;

- Decúbito elevado (fowler 60);

- Mobilização ativo-assistida de MMSS* e MMII** (2x ao dia com 15 repetições - de acordo com a identificação de déficit que o paciente obtiver na avaliação).

PROTOCOLO

NÍVEL 1

$\rightarrow$ diagonais funcionais de MMSS; flexão-extensão de cotovelo; flexão-extensão de punho; tríplice flexão de MMIl; exercícios metabólicos de extremidades; descarga de peso em MMII.

- Propriocepção articular;

- Alongamento estático;

- Estimulação elétrica neuromuscular.

Perme: 7-11 pontos (Paciente acordado e alerta)

- Posicionamento;

- Decúbito elevado (fowler 60);

PROTOCOLO - Mobilização ativa de MMSS e MMII (idem ao nível 1);

NÍVEL 2

- Sedestação fora do leito pelo menos 20 minutos - passiva ou ativa assistida.

- Propriocepção articular;

- Alongamento estático;

- Estimulação elétrica neuromuscular (30 minutos, largura de pulso (ms) 0,35, frequência $(\mathrm{Hz}) 35$, intensidade: contração visível);

Perme: 12-21 pontos (Paciente alerta e consciente, com MRC de MMSS e MMII $\geq 3$ )

- Posicionamento;

- Decúbito elevado (fowler $60^{\circ}$ ) ou ortostatismo assistido e/ou ativo;

PROTOCOLO

- Mobilização aeróbica ativa de MMSS e MMII (2 x ao dia com 15 repetições - de acordo com a identificação de déficit

- Sedestação no leito sem apoio pelo menos 20 minutos. Sedestação na poltrona com assistência (20 min/dia);

- Transferência do paciente para beira leito (exercícios de controle de tronco e equilíbrio).

- Propriocepção articular;

- Alongamento estático;

- Cicloergômetro (2x ao dia - manhã e tarde - 15 minutos, carga progressiva diariamente).

Perme: 22-32 pontos (Paciente com MRC de MMSS e MMII $\geq 4$ )

- Posicionamento;

- Decúbito elevado (fowler $60^{\circ}$ ) ou ortostatismo ativo;

PROTOCOLO

- Treino de força com exercícios resistidos de MMSS e MMII: agachamento, push-up, ponte ( 2 x ao dia com 15

NÍVEL 4

repetições - de acordo com a identificação de déficit que o paciente obtiver na avaliação);

- Transferência ativa ou com mínima assistência para a poltrona (no mínimo 20min/dia);

- Treino de ortostatismo;

- Deambulação;

- Alongamento estático;

- Cicloergômetro (idem ao nível 3).

*MMSS: Membros Superiores; **MMII: Membros Inferiores. 
redução do grau de dependência do doente, melhorando o seu desempenho nas atividades de vida diária. Uma boa terapêutica é o treino de tronco, exercício essencial para fornecer melhora na estabilização e mobilidade do paciente, ao mesmo tempo que trabalha o equilíbrio e a capacidade de se manter ereto, corroborando com a restauração da funcionalidade global e consequentemente com a sua melhor recuperação 20,21 .

A sedestação deve se fazer presente durante todo o período de tratamento, sendo realizada de forma passiva ou ativa, tem como objetivo otimizar o transporte de oxigênio e a troca gasosa, essa atividade melhora o estado de alerta do paciente, além de facilitar uma boa resposta antigravitacional e promover reexpansão pulmonar, reduzindo os efeitos da imobilidade e do repouso, proporcionando conforto ao paciente, redução do tempo de internação e melhor qualidade de vida durante e após hospitalização ${ }^{6}$. Deve ser realizada assim que possível para trabalhar propriocepção, equilíbrio estático e dinâmico, força muscular principalmente de tronco e pescoço ${ }^{22}$. Ao sentar o paciente no leito ou cadeira estamos realizando uma transferência importante para chegar ao ortostatismo e a marcha, além de que o posicionamento sentado pode contribuir para a realização de cinesioterapia motora, aumentando a força dos músculos em geral ${ }^{23-25}$.

Um escore de 12 a 21 pontos indica um paciente alerta e consciente com MRC de membros superiores e membros inferiores $\geq 3$, que necessita de mínima assistência ${ }^{7}$. A graduação de força muscular em pacientes internados é fundamental para o direcionamento das condutas fisioterapêuticas de mobilização, bem como para quantificar a evolução e respostas diante as intervenções adotadas, as reavaliações irão colaborar para quantificar a recuperação deste paciente ${ }^{26}$.

Existem recursos para auxiliar na mobilização precoce e ganho de força, um exemplo comum é o cicloergômetro, recurso que pode ser utilizado ainda no leito ou na poltrona. Assim como os exercícios ativos e com resistência são seguros e eficazes para recuperar e/ou ganhar performance funcional e força ${ }^{27}$. Um estudo ${ }^{28}$ constatou que o uso desse dispositivo numa frequência de 4 sessões por semana com 20 minutos e ajuste da intensidade conforme força muscular do paciente, melhorou a fraqueza muscular, a tolerância ao treinamento físico, a dispneia e dessaturação, corroborando com a diminuição no tempo de internação ${ }^{28}$.

A prática terapêutica através da posição em ortostase é bastante encorajada em doentes com base em seus benefícios, que incluem melhora do controle autonômico do sistema cardiovascular, melhora da oxigenação, aumento da ventilação, melhora do estado de alerta, estimulação vestibular, facilitação de resposta postural antigravitacional, prevenção de contraturas articulares e úlceras por pressão ${ }^{29}$.

O paciente que obtiver de 22 a 32 pontos na Escala Perme é um indivíduo com MRC de MMSS e MMII $\geq 4$, mais independente e que não necessita de assistência ${ }^{7}$, é um indivíduo em que se deve concentrar o tratamento no ganho de força e funcionalidade, assim oferecendo um melhor retorno a vida social, pois é um candidato à alta hospitalar. Terapêuticas com o máximo de autonomia e independência devem ser as principais abordadas nesta fase da hospitalização. A deambulação, treino de equilíbrio e força são exercícios que devem ser exercitados, pois refletem diretamente na condição funcional dos pacientes doentes em sua alta, favorece a recuperação durante a internação e a após a alta, assim como reflete diretamente no retorno às atividades de vida diária da melhor forma e o mais precoce possível ${ }^{30}$.

O exercício gera efeitos agudos e crônicos, como redução da incapacidade funcional, da intensidade dos pensamentos negativos decorrentes das doenças físicas; e promoção da melhoria do bem estar e do humor. Os fisioterapeutas dentro do hospital devem assumir a responsabilidade de estimular o paciente a prática do exercício físico durante e após hospitalização, ajudando-os a ser integrado à sociedade, físico e mentalmente ativo, apto para desenvolver com liberdade as tarefas do seu cotidiano após a alta hospitalar ${ }^{31}$.

\section{CONCLUSÃO}

Avaliações periódicas e reavaliações são de grande importância para quantificar a recuperação do paciente frente às estratégias adotadas no tratamento fisioterapêutico. A mobilização precoce realizada através de um instrumento norteador como o protocolo está associada a melhores resultados funcionais, devendo ser realizada sempre que indicada, respeitando as contraindicações, limitações e variações biológicas nos adultos.

A escala Perme e o protocolo de exercício são instrumentos de grande potencial e devem ser utilizados durante todo o período de hospitalização, porém existem poucos estudos na literatura referente a esses temas, dito isto, outros estudos devem ser feitos a fim de ampliar conhecimento e quantificar maior número de evidências e resultados.

\section{FONTE DE FINANCIAMENTO}

Nada a declarar.

\section{CONFLITO DE INTERESSES}

Nada a declarar.

\section{REFERÊNCIAS}

1. Machado AS, Nunes RD, Rezende AAB. Intervenções fisioterapêuticas para mobilizar precocemente os pacientes internados em unidades de terapia intensiva: estudo de revisão. Rev Amazonia Sci Health. 2016 Abr/Jun;4(2):41-6. http://dx.doi.org/10.18606/2318-1419/amazonia.sci.health. v4n2p41-46.

2. Mota CM, Silva VG. A segurança da mobilização precoce em pacientes críticos: uma revisão de literatura. Rev Interfaces 
Cient Saúde Ambiente. 2012;1(1):83-91. http://dx.doi. org/10.17564/2316-3798.2012v1n1p83-91.

3. Nacer RS. Novos paradigmas da abordagem fisioterapêutica na unidade de terapia intensiva: mobilização precoce no paciente crítico. Interbio. 2012;6(1).

4. Gosselink R, Bott J, Johnson M, Dean E, Nava S, Norrenberg $M$, et al. Physiotherapy for adult patients with critical illness: recommendations of the European Respiratory Society and European Society of Intensive Care Medicine Task Force on Physiotherapy for Critically III Patients. Intensive Care Med. 2008;34(7):1188-99. http://dx.doi.org/10.1007/s00134-0081026-7. PMid:18283429.

5. Morris PE, Goad A, Thompson C, Taylor K, Harry B, Passmore L, et al. Early intensive care unit failure. Crit Care Med. 2008;36(8):2238-43. http://dx.doi.org/10.1097/ CCM.0b013e318180b90e. PMid:18596631.

6. França EÉ, Ferrari F, Fernandes P, Cavalcanti R, Duarte A, Martinez BP, et al. Fisioterapia em pacientes críticos adultos: recomendações do Departamento de Fisioterapia da Associação de Medicina Intensiva Brasileira. Rev Bras Ter Intensiva. 2012;24(1):6-22. PMid:23917708.

7. Perme C, Nawa RK, Winkelman C, Masud F. A tool to assess mobility status in critically ill patients: the Perme Intensive Care Unit Mobility Score. Methodist DeBakey Cardiovasc J. 2014 Jan-Mar;10(1):41-9. http://dx.doi.org/10.14797/mdcj10-1-41. PMid:24932363.

8. Kawaguchi VM, Pires No RC. Alterações hemodinâmicas durante alteração de exercícios em pacientes críticos. Programa de atualização em fisioterapia em terapia intensiva adulto. PROFISIO. 2015;5(3):87-110.

9. Dubb R, Nydahl P, Hermes C, Schwabbauer N, Toonstra A, Parker AM, et al. Barriers and strategies for early mobilization of patientsin intensive care units. Ann Am Thorac Soc. 2016 May;13(5):724-30. http://dx.doi. org/10.1513/AnnalsATS.201509-586CME. PMid:27144796.

10. Aquim EE, Bernardo WM, Buzzini RF, Azeredo NSG, Cunha LSD, Damasceno MCP, et al. Diretrizes brasileiras de mobilização precoce em unidade de terapia intensiva. Rev Bras Ter Intensiva. 2019;31(4):434-43. http://dx.doi. org/10.5935/0103-507X.20190084. PMid:31967216.

11. Guimarães FS, Martins JA. Assobrafir Profisio: fisioterapia em terapia intensiva no adulto. Porto Alegre: Panamericana; 2010. (Ciclo 1, módulo 1).

12. Hermans $G$, van den Berghe $G$. Clinical review: intensive care unit acquired weakness. Crit Care Trop. 2015 Oct;19(1):1-9.

13. Monteiro N, Guedes G, Rocha Jr A. Análise da qualidade de vida dos pacientes internados em âmbito hospitalar: importância da Fisioterapia. Rev Interdisciplin Estud Exp. 2009;1(4):7-12.

14. Pinheiro A, Christofoletti G. Fisioterapia motora em pacientes internados na unidade de terapia intensiva: uma revisão sistemática. Rev Bras Ter Intensiva. 2012;24(2):18896. http://dx.doi.org/10.1590/S0103-507X2012000200016. PMid:23917769.

15. Clini E, Ambrosino N. Early physiotherapy in the respiratory intensive care unit. Respir Med. 2005;99(9):1096-104. http:// dx.doi.org/10.1016/j.rmed.2005.02.024. PMid:16085211.

16. Stevens RD, Marshall SA, Cornblath DR, Hoke A, Needham DM, De Jonghe B, et al. A framework for diagnosing and classifying intensive care unit-acquired weakness. Crit Care Med. 2009;37(10, Supl. 10):299-308. http://dx.doi. org/10.1097/CCM.0b013e3181b6ef67. PMid:20046114.

17. Martinez BP, Bispo AO, Duarte ACM, Mansueto GN. Declínio funcional em uma unidade de terapia. Rev Inspirar Mov Saúde. 2013;5(1):1-5.
18. Norrenberg M, Backer D, Moraine JJ. Oxygen consumption can increase during passive leg mobilization. Intensive Care Med. 1995;21(Supl):S177.

19. Borges VM, Oliveira LRC, Peixoto E, Carvalho NAA. Fisioterapia motora em pacientes adultos em terapia intensiva. Rev Bras Ter Intensiva. 2009;21(4):446-52. http://dx.doi.org/10.1590/ S0103-507X2009000400016. PMid:25307338.

20. Jung K-S, Cho H-Y, In T-S. Trunk exercises performed on an unstable surface improve trunk muscle activation, postural control, and gait speed in patients with stroke. J Phys Ther Sci. 2016;28(3):940-4. http://dx.doi.org/10.1589/jpts.28.940. PMid:27134389.

21. Castaneda L, Bergmann A, Bahia L. A Classificação Internacional de Funcionalidade, Incapacidade e Saúde: uma revisão sistemática de estudos observacionais. Rev Bras Epidemiol. 2014;17(2):437-51. http://dx.doi. org/10.1590/1809-4503201400020012ENG. PMid:24918415.

22. Buttignol M, Pires No RC, Annoni R. Protocolos de mobilização precoce no paciente crítico: up-to-date. In: Associação Brasileira de Fisioterapia Cardiorrespiratória e Fisioterapia em Terapia Intensiva, editor. PROFISIO ciclo 7. Porto Alegre: Artmed Panamericana; 2016. p. 61-101. (Sistema de Educação Continuada a Distâncias, vol. 2).

23. Silva IT, Oliveira AA. Efeitos da mobilização precoce em pacientes críticos internados em UTI. C\&D Rev Eletrôn Fainor. 2015;8(2):41-50.

24. Nielsen KG, Holte $K$, Kehlet $H$. Effects of posture on postoperative pulmonary function. Acta Anaesthesiol Scand. 2003;47(10):1270-5. http://dx.doi.org/10.1046/j.13996576.2003.00240.x. PMid:14616326.

25. Hickmann $\mathrm{CH}$, Montecinos-Munoz RN, Castanares-Zapatero D, Arriagada-Garrido RS, Jeria-Blanco U, Gizzatullin T, et al. Acute effects of sitting out of bed and exercise on lung aeration and oxygenation in critically III subjects. Respir Care. 2021;66(2):253-62. http://dx.doi.org/10.4187/ respcare.07487. PMid:32994357.

26. Lee JJ, Waak K, Grosse-Sundrup M, Xue F, Lee J, Chipman $D$, et al. Global Muscle strength but not grip strength predicts mortality and length of stay in a general population in a surgical intensive care unit. Phys Ther. 2012;92(12):1546-55. http://dx.doi.org/10.2522/ptj.20110403. PMid:22976446.

27. Santos F, Mandelli PGB, Ostrowski VR, Tezza R, Dias JS. Relação entre a mobilização precoce e tempo de internação em uma unidade de terapia intensiva. Rev Gestão \& Saúde. 2015;6(2):1394-07. http://dx.doi.org/10.18673/ gs.v6i2.22475.

28. Burtin C, Clerckx B, Robbeets C, Ferdinande P, Langer D, Troosters $\mathrm{T}$, et al. Early exercise in critically ill patients anhances short-term functional recovery. Crit Care Med. 2009;37(9):2499-505. http://dx.doi.org/10.1097/ CCM.0b013e3181a38937. PMid:19623052.

29. Vellar CM, Forti G. Ortostatismo passivo em pacientes comatosos na UTI - um estudo preliminar. Rev Neurociênc. 2008;16(1):16-9.

30. Schweickert WD, Pohlman MC, Pohlman AS, Nigos C, Pawlik AJ, Esbrook CL, et al. Early physical and occupational therapy in mechanically ventilated, critically ill patients: a randomised controlled trial. Lancet. 2009;373(9678):187482. http://dx.doi.org/10.1016/S0140-6736(09)60658-9. PMid:19446324.

31. Bossi CL. A evolução dos exercícios resistido. Sprint Mag. 2003;(126):23-5. 